\title{
Symbolic Reasoning for Automatic Signal Placement
}

\author{
Kostas Ferles \\ The University of Texas at Austin, USA \\ kferles@cs.utexas.edu \\ Isil Dillig \\ The University of Texas at Austin, USA \\ isil@cs.utexas.edu
}

\author{
Jacob Van Geffen \\ The University of Texas at Austin, USA \\ jsv@cs.utexas.edu \\ Yannis Smaragdakis \\ University of Athens, Greece \\ smaragd@di.uoa.gr
}

\begin{abstract}
Explicit signaling between threads is a perennial cause of bugs in concurrent programs. While there are several runtime techniques to automatically notify threads upon the availability of some shared resource, such techniques are not widely-adopted due to their run-time overhead. This paper proposes a new solution based on static analysis for automatically generating a performant explicit-signal program from its corresponding implicit-signal implementation. The key idea is to generate verification conditions that allow us to minimize the number of required signals and unnecessary context switches, while guaranteeing semantic equivalence between the source and target programs. We have implemented our method in a tool called Expresso and evaluate it on challenging benchmarks from prior papers and open-source software. ExPRESSo-generated code significantly outperforms past automatic signaling mechanisms (avg. 1.56x speedup) and closely matches the performance of hand-optimized explicit-signal code.
\end{abstract}

CCS Concepts - Software and its engineering $\rightarrow$ Concurrent programming languages; Concurrent programming structures;

Keywords Implicit signal monitors, concurrent programming, symbolic reasoning, verification conditions, abductive reasoning, monitor invariant

\section{ACM Reference Format:}

Kostas Ferles, Jacob Van Geffen, Isil Dillig, and Yannis Smaragdakis. 2018. Symbolic Reasoning for Automatic Signal Placement. In Proceedings of 39th ACM SIGPLAN Conference on Programming Language Design and Implementation (PLDI'18). ACM, New York, NY, USA, 15 pages. https://doi.org/10.1145/3192366.3192395

Permission to make digital or hard copies of all or part of this work for personal or classroom use is granted without fee provided that copies are not made or distributed for profit or commercial advantage and that copies bear this notice and the full citation on the first page. Copyrights for components of this work owned by others than the author(s) must be honored. Abstracting with credit is permitted. To copy otherwise, or republish, to post on servers or to redistribute to lists, requires prior specific permission and/or a fee. Request permissions from permissions@acm.org. PLDI'18, fune 18-22, 2018, Philadelphia, PA, USA

(c) 2018 Copyright held by the owner/author(s). Publication rights licensed to Association for Computing Machinery.

ACM ISBN 978-1-4503-5698-5/18/06 ..\$15.00

https://doi.org/10.1145/3192366.3192395

\section{Introduction}

A common challenge in concurrent programming is to coordinate access to shared resources and achieve correct synchronization between different threads. While there are many different language constructs that can be used to perform synchronization, a widely-established programming pattern is to encapsulate inter-thread coordination using monitors $[24,29,40]$. At a high level, a monitor encapsulates all shared state between threads and guarantees mutual exclusion. In addition, monitors perform synchronization between threads by blocking and unblocking them depending on the availability of some shared resource.

Broadly speaking, monitors can be classifed into two categories, depending on the burden they impose on the system vs. the programmer [7]. In particular, explicit-signal monitors typically employ condition variables to perform synchronization between threads and use an explicit "signal" construct to notify other threads when some shared resource becomes available. In contrast, implicit-signal (automatic) monitors provide a waituntil( $P$ ) construct such that any thread executing this statement blocks until predicate $P$ becomes true. In implicit-signal monitors, there is no explicit signal construct, and it is the responsibility of the system to notify threads that are currently blocked on a predicate. To give the reader some intuition, Figure 1 shows the implementation of an implicit-signal monitor for the well-known readerswriters problem, and Figure 2 shows its corresponding implementation as an explicit-signal monitor.

As illustrated by the example from Figures 1 and 2, programming with implicit monitors is considerably easier because the programmer does not need to reason about when and which threads should be notified. In fact, it is well-known that many concurrency bugs are caused by erroneous signal placement in explicit-signal implementations [23, 32]. However, despite their easier programmability, implicit-signal monitors are not widely-used due to performance considerations. In particular, because the system needs to notify threads that are blocked on a predicate, run-time support for implicit-signal monitors may result in considerable overhead. For example, according to Buhr et al., automatic monitors can be 10-50 times slower than explicit signals [7]. Even though recent work by Hung and Garg proposes a more efficient implementation of automatic monitors [31], explicit-signal 
monitors still remain the de-facto synchronization mechanism in real-world concurrent programs.

In this paper, we propose a new solution - based on static analysis - to programming with implicit-signal monitors. Given the implementation of an implicit-signal monitor, our method automatically synthesizes an efficient and semantically equivalent explicit-signal implementation. We believe this approach has two advantages compared to prior runtime techniques: First, because our method does not require additional run-time book-keeping, it has the potential to be as efficient as a performant hand-written explicit-signal implementation. Second, because the code generated by our system can be inspected and further refined by the programmer, it is more transparent compared to automatic-signaling systems that provide run-time instrumentation.

While it is straightforward to generate any semantically equivalent explicit-signal implementation of an automaticsignal monitor, a key consideration is the efficiency of the synthesized code. In particular, the synthesized code should not spuriously wake up threads that are blocked on a predicate that evaluates to false. ${ }^{1}$ In practice, this means that the generated code should not notify threads blocked on a predicate $P$, if $P$ is guaranteed to be false at the time of notification. Furthermore, whenever possible, the generated code should notify a single -rather than all-threads blocked on a predicate in order to avoid unnecessary context switches.

In addition to avoiding spurious wake-ups, another important efficiency consideration is to minimize the use of conditional signals, which notify other threads only if some condition evaluates to true. Because conditional signals require evaluating the truth value of (potentially complex) predicates at run-time, it is desirable to use unconditional signals whenever possible. In fact, while some run-time solutions, such as AutoSynch [31] avoid spurious wake-ups altogether, they may still incur significant overhead due to the frequent evaluation of predicates at run-time.

The solution that we adopt in this paper tries to minimize both the use of spurious wake-ups as well as conditional signals by performing precise static analysis of the monitor code. In particular, our method automatically generates Hoare triples, that, if valid, allow us to establish that a program fragment does not need to signal other threads waiting on a predicate. For program fragments where signaling may be necessary, our method generates additional Hoare triples whose validity allows us to minimize the use of conditional signals as well as broadcast operations that notify all threads.

In order to successfully discharge the generated Hoare triples, our method uses so-called monitor invariants, which are assertions that hold every time a thread enters or leaves

\footnotetext{
${ }^{1}$ While a thread that is spuriously woken up will "go back to sleep", this introduces significant overhead due to an unnecessary context-switch.
}

the monitor. Our approach automatically infers these monitor invariants by combining abductive reasoning and predicate abstraction, allowing the synthesis of non-trivial invariants that involve disjunctions. Monitor invariants allow us to discharge verification conditions that could not be proven otherwise (e.g., by strengthening the precondition of the generated Hoare triples) and are therefore crucial for generating efficient explicit-signaling code.

We have implemented our proposed ideas in a tool called ExPRESSO and evaluate the efficiency of the code generated by ExPREsso by comparing it against manually written explicit-signal monitors as well as the state-of-the-art AutoSynch tool that provides run-time support for implicitsignal monitors. Our evaluation shows that the performance of the code synthesized by ExPRESSo is an average of 1.56x faster than AutoSynch and comparable to that of handwritten code.

In all, this paper makes the following key contributions:

- We propose a novel technique, based on static analysis, for generating efficient explicit-signal implementations of implicit-signal monitors.

- We show how the automatic signal placement problem can be reduced to proving the validity of certain kinds of Hoare triples in concurrent programs.

- We introduce the notion of monitor invariants and show how to automatically infer them using abductive reasoning and monomial predicate abstraction.

- We implement the proposed techniques in a tool called ExPRESSO and evaluate it by comparing against AutoSynch, a state-of-the art runtime system for implicit-signal monitors, as well as hand-written code.

\section{Overview of Technique}

In this section, we give a high-level overview of our approach with the aid of the reference Readers-Writers example, shown in Figure 1. In particular, we explain the reasoning performed by ExpREsso to automatically generate the code shown in Figure 2 by analyzing the implicit-signal monitor of Figure 1.

EXPRESSO starts its analysis by inferring a monitor invariant, which is an assertion that holds every time a thread enters or exits the monitor. For the code in Figure 1, ExPRESSO successfully infers the invariant readers $\geq 0$. Then, ExPRESSo uses this invariant to determine for each conditional critical section in Figure 1 (a) if signaling is necessary, (b) whether to signal or broadcast, and (c) whether to do so conditionally or unconditionally.

EnterReader. Consider a reader thread $t_{r}$ executing the method enterReader. To generate explicit signaling code, we need to determine whether $t_{r}$ needs to notify any writer threads blocked on predicate $P_{w}=($ readers $=0 \wedge \neg$ writerIn $)$ at line 13. Towards this goal, we ask the following question: "Assuming that a writer thread $t_{w}$ is blocked on $P_{w}$, is it possible that $P_{w}$ becomes true after $t_{r}$ executes the code in 


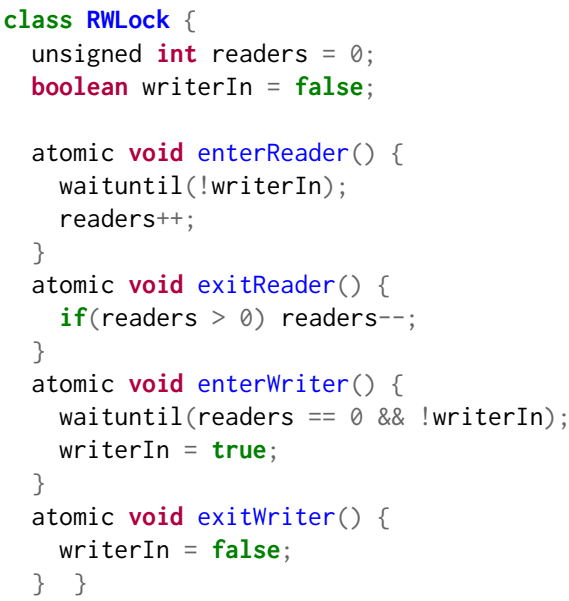

Figure 1. Implicit-signal monitor for readers-writers lock.

enterReader?". If the answer to this question is "no", we have established that $t_{r}$ does not need to signal. Thus, to prove that no signals are necessary, ExPRESSO generates and checks the validity of the following Hoare triple:

$$
\left\{\text { readers } \geq 0 \wedge \neg \text { writerIn } \wedge \neg P_{w}\right\} \text { readers++ }\left\{\neg P_{w}\right\}
$$

Here, the precondition states that (a) the monitor invariant holds when $t_{r}$ enters the monitor, (b) ! writerIn must hold if $t_{r}$ executes readers++, and (c) $P_{w}$ is false, meaning that some writer thread may be blocked at line 13 . The post-condition says that $P_{w}$ continues to stay false after $t_{r}$ exits the monitor. Since this Hoare triple is indeed valid, ExPREsso establishes that no signaling is necessary. Observe that dropping the conjunct readers $\geq 0$ from the precondition would result in a Hoare triple that is not valid; thus, the monitor invariant is crucial for avoiding the signal operation in this example.

ExitReader. For the exitReader method, ExPREsso needs to determine whether we should signal any reader threads blocked at line 6 or writer threads blocked at line 13. Using similar reasoning as in enterReader, it is easy to establish that we do not need to signal reader threads because readers-does not affect the truth value of the predicate writerIn. Now, to determine the necessity of signaling writer threads, EXPRESSO generates the following Hoare triple:

$\left\{\right.$ readers $\left.\geq 0 \wedge \neg P_{w}\right\}$ if $($ readers $>0)$ readers-- $\left\{\neg P_{w}\right\}$

Since this Hoare triple is not valid, signalling is necessary.

Next, Expresso tries to determine whether it suffices to notify a single writer thread or we need to notify all writers blocked at line 13. To answer this question, we ask "Is it possible that $P_{w}$ stays true after some writer thread $t_{w}$ executes enterWriter?". If not, we have proven that it is unnecessary (and wasteful) to wake up multiple threads, since $P_{w}$ becomes false after the first writer thread executes. Thus, EXPRESSO generates and checks the following Hoare triple:

$$
\left\{\text { readers } \geq 0 \wedge P_{w}\right\} \text { writerIn }=\text { true }\left\{\neg P_{w}\right\}
$$

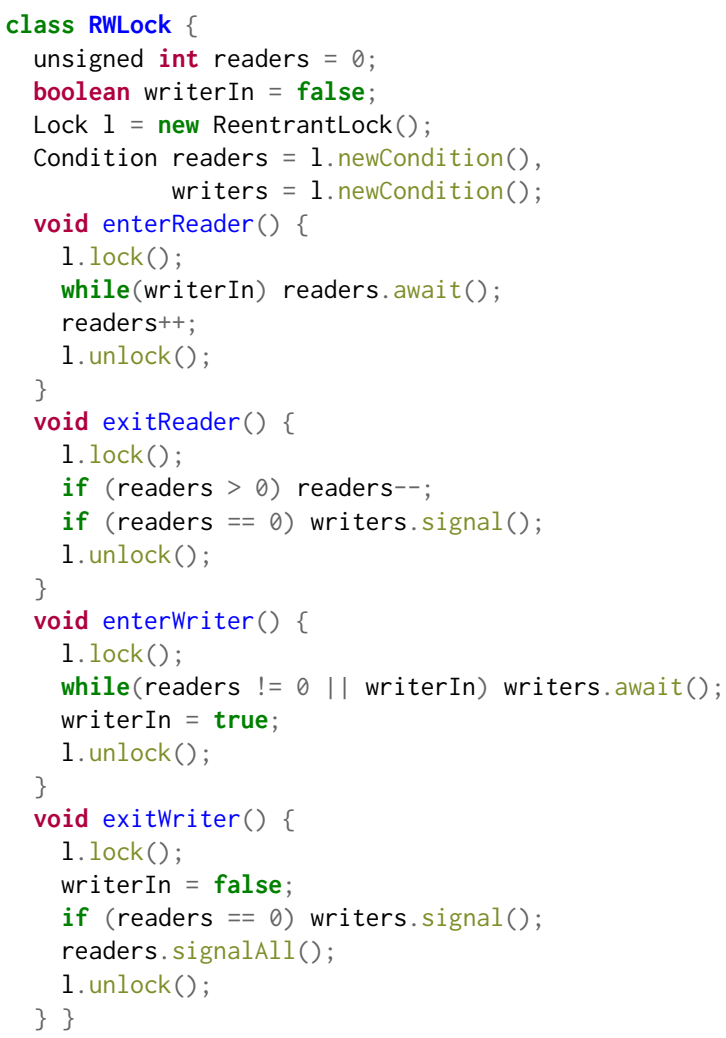

Figure 2. Explicit-signal monitor for readers-writers lock.

Since this triple is valid, Expresso has determined that broadcasting is not necessary.

Finally, EXPREsso checks whether it can signal unconditionally, meaning that $P_{w}$ is guaranteed to hold after the reader thread $t_{r}$ exits the monitor. Towards this goal, we perform the following check:

$$
\left\{\text { readers } \geq 0 \wedge \neg P_{w}\right\} \text { if }(\text { readers }>0) \text { readers-- }\left\{P_{w}\right\}
$$

This Hoare triple is not valid, so ExPREsso signals conditionally in order to avoid a spurious wake-up.

EnterWriter. Using similar reasoning as in enterReader, ExPRESSO can establish that enterWriter does not need to signal any readers because the following Hoare triple is valid:

$\left\{\right.$ readers $\geq 0 \wedge P_{w} \wedge$ writerIn $\}$ writer In $=$ true $\{$ writerIn $\}$

ExitWriter. For exitWriter, ExPRESSO establishes that it is necessary to notify both reader and writer threads. Using similar reasoning as in exitReader, we can prove that broadcasting for all writer threads is not necessary, however, we need to perform conditional signaling to avoid spurious wake-ups. For reader threads, ExpREsso determines that broadcasting is necessary since !writer In continues to hold after executing the statement readers++. Furthermore, since the Hoare triple

$\{$ readers $\geq 0 \wedge$ writerIn $\}$ writer $I n=$ false $\{\neg$ writer $I n\}$ 
is valid, ExPREsso can establish that ! writerIn must be true after the writer thread exits. Thus, Expresso instruments the code to signal reader threads unconditionally.

Summary. For this example, the code generated by ExPRESSO is precisely the same one as the human-written explicit-signal implementation shown in Figure 2. Observe that ExPRESSO can prove the gratuitousness of broadcasts, and it can also establish that enterReader and enterWriter do not need to signal. Finally, note that some of the Hoare triples generated by ExPREsso could not be established without the useful monitor invariant readers $\geq 0$.

\section{Source and Target Languages}

In this section, we present some preliminary concepts related to concurrent programming and describe the source and target languages that can be used to implement implicit- and explicit-signal monitors respectively. The goal of the two languages presented here is to provide a unified theoretical framework suitable for automatic reasoning. In Section 6, we discuss how the target language can be instantiated in a concrete monitor implementation.

\subsection{Preliminaries}

In this paper, we consider a shared-memory concurrency model in which all accesses to shared resources occur inside a monitor. In other words, all variables accessed outside the monitor are assumed to be thread-local. We represent threads using integer identifiers drawn from the set $T \subseteq \mathbb{N}$. Because we do not impose any restrictions on the number of threads that can execute monitor code, our approach is applicable to parametrized concurrent programs.

We partition program variables used in the monitor into two disjoint sets, namely $L$ and $G$, representing thread-local and shared (global) variables respectively. As stated by the definition below, the state $\sigma$ of a monitor identifies the values of program variables for each thread.

Definition 3.1. (Monitor state) A monitor state, $\sigma: T \times$ $(L \cup G) \rightarrow \mathbb{N}$, is a mapping from (thread identifier, monitor variable) pairs to a value. We require monitor states to agree on the values of shared variables for all threads; i.e.,

$$
\forall t_{1}, t_{2} \in T, v \in G . \sigma\left(t_{1}, v\right)=\sigma\left(t_{2}, v\right)
$$

\subsection{Source Language}

Because our approach transforms an automatic-signal monitor to an explicit-signal one, we first present the source language in which automatic-signal monitors are implemented.

The syntax of our source language is presented in Figure 3. Since our implementation targets Java programs, we consider implicit-signal monitors written in a simple objectoriented language with Java-like syntax. In particular, an automatic-signal monitor consists of a set of field declarations and a set of atomic methods - i.e., the body of a method $m$ executes without interruption unless the thread blocks on

$$
\begin{aligned}
& \text { Monitor } M \quad::=\text { monitor } M\left\{(\text { fld } \mid m)^{*}\right\} \\
& \text { Field fld } \quad::=f: \tau \\
& \text { Method } m \quad::=\text { atomic } m(\vec{v})\left\{w^{*} \text {; return } v ;\right\} \\
& \text { WUntil } w \quad::=\text { waituntil }(p)\{s\} \\
& \text { Statement } s \quad::=\operatorname{skip}\left|s_{1} ; s_{2}\right| v:=e \mid v \cdot f:=e \\
& \text { if }(p)\left\{s_{1}\right\} \text { else }\left\{s_{2}\right\} \\
& \text { while }(p)\{s\}
\end{aligned}
$$

Figure 3. Implicit-signal monitor language. Here, $e$ and $p$ denote expressions and predicates respectively.

some waituntil statement whose corresponding predicate evaluates to false. To simplify presentation, we will assume that local variables of different methods have unique names.

The body of each monitor method is a sequence of statements of the form waituntil $(p)\{s\}$, where $p$ is a predicate and $s$ is a statement (assignment, store, sequence, loop etc.). Observe that a statement $s$ is a special case of a waituntil statement whose corresponding predicate is true. We refer to predicate $p$ as the guard of the waituntil construct and to statement $s$ as its body and sometimes write $w=(p, s)$ to denote a waituntil statement with guard $p$ and body $s$. Given a monitor $M$, we use the notation $C C R s(M)$ to represent the set of all waituntil statements used in any method in $M$.

While waituntil statements can only appear as top-level statements in our source language, we note that this design decision does not sacrifice expressiveness. For example, consider the code snippet if (c) waituntil(p), which is not supported by our source language. Observe that the check if (c) can be moved outside of the monitor if $c$ is not on shared data. On the other hand, if $c$ does involve shared data, the condition is either checked in a logically racy way ${ }^{2}$ or the programmer knows that c cannot change while the thread is blocked on p. In either case, the program's logic is preserved or enhanced if condition $c$ is moved inside the waituntil statement.

In the rest of this paper, we assume the standard semantics of waituntil $(p)\{s\}$ statements where a thread $t$ atomically performs the following actions: It first evaluates the boolean predicate $p$. If $p$ evaluates to true, $t$ also executes $s$ immediately after the evaluation of $p$. Otherwise, $t$ is blocked until $p$ becomes true.

Since the semantics of statements $s$ are standard, we do not present them in detail and use the notation $\langle s, t, \sigma\rangle \Downarrow \sigma^{\prime}$ to indicate the resulting monitor state $\sigma^{\prime}$ when thread $t$ executes statement $s$ under initial state $\sigma$. Given a monitor state $\sigma$, thread $t$, and predicate $p$, we write $(\sigma, t) \mid=p$ if $p$ evaluates to true and $(\sigma, t) \not \neq p$ if $p$ evaluates to false.

Monitor traces. To define the semantics of monitors, we first introduce the notion of a monitor trace. A monitor trace $\tau$ is a sequence of monitor events where each event $e$ is a triple $(t, w, b)$ where $t$ is a thread identifier, $w$ is a

\footnotetext{
${ }^{2}$ i.e., condition c could have changed while the thread is waiting on $p$
} 


$$
\begin{aligned}
& e=(t, w, \text { true }) \\
& \bar{e} \notin \mathcal{B} \quad(\sigma, t) \mid=\operatorname{Guard}(w) \\
& \langle\operatorname{Body}(w), t, \sigma\rangle \Downarrow \sigma^{\prime} \\
& \text { (2a) } \frac{\mathcal{N}^{\prime}=\left\{(t, w)\left|(t, w) \in \mathcal{B},\left(\sigma^{\prime}, t\right)\right|=\operatorname{Guard}(w)\right\}}{(\sigma, e, \mathcal{B}, \mathcal{N}) \longrightarrow\left(\sigma^{\prime}, \epsilon, \mathcal{B}, \mathcal{N} \cup \mathcal{N}^{\prime}\right)} \\
& e=(t, w, \text { true }) \\
& \bar{e}=\min (\mathcal{N}) \quad(\sigma, t) \mid=\operatorname{Guard}(w) \\
& \langle\operatorname{Body}(w), t, \sigma\rangle \Downarrow \sigma^{\prime} \\
& \frac{\mathcal{N}^{\prime}=\left\{(t, w)\left|(t, w) \in \mathcal{B},\left(\sigma^{\prime}, t\right)\right|=\operatorname{Guard}(w)\right\}}{(\sigma, e, \mathcal{B}, \mathcal{N}) \longrightarrow\left(\sigma^{\prime}, \epsilon, \mathcal{B} \backslash\{\bar{e}\},\left(\mathcal{N} \cup \mathcal{N}^{\prime}\right) \backslash\{\bar{e}\}\right)} \\
& \frac{(\sigma, e, \mathcal{B}, \mathcal{N}) \longrightarrow\left(\sigma^{\prime}, \epsilon, \mathcal{B}^{\prime}, \mathcal{N}^{\prime}\right)}{(\sigma, e:: \tau, \mathcal{B}, \mathcal{N}) \longrightarrow\left(\sigma^{\prime}, \tau, \mathcal{B}^{\prime}, \mathcal{N}^{\prime}\right)}
\end{aligned}
$$

Figure 4. Transition relation for implicit-signal monitor traces. Given an event $e=(t, w, b), \bar{e}$ denotes $(t, w)$. We assume there is a total order relation $\prec$ between events and min picks the minimum one with respect to $\prec$.

waituntil statement, and $b$ is a boolean indicating whether the guard of $w$ evaluates to true or false. In particular, the event $(t, w$, false $)$ indicates that thread $t$ was blocked on the guard of $w$, whereas the event $(t, w$, true) indicates that $t$ was able to execute $w$ in its entirety. Given an event $e=(t, w, b)$, we write $\bar{e}$ to denote the pair $(t, w)$.

We say that a monitor trace is syntactically well-formed if it (a) respects the relative ordering of statements within a method, (b) obeys the requirement that a thread cannot execute method $m^{\prime}$ before finishing the execution of method $m$, and (c) satisfies the invariant that a thread exits the monitor either by blocking on a predicate or by finishing the execution of a method. A more formal definition of syntactic well-formedness is given in the extended version of the paper [21].

Example 3.2. Consider the following monitor $M$, where we elide the "atomic" keywords for brevity:

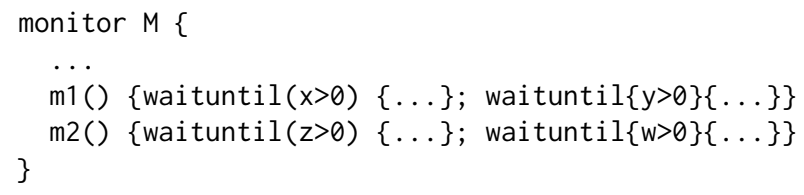

Let us refer to the $j$ 'th waituntil statement in method $i$ as $w_{i j}$. The trace $\left[\left(1, w_{12}\right.\right.$, true $),\left(1, w_{11}\right.$, true $\left.)\right]$ is not syntactically well-formed since the same thread cannot execute $w_{12}$ before $w_{11}$ (i.e., it violates requirement (a)). Similarly, the trace $\left[\left(1, w_{11}\right.\right.$, false $),\left(1, w_{21}\right.$, true $\left.)\right]$ is also not syntactially well-formed since the same thread cannot execute method

$$
\begin{aligned}
& e=(t, w, \text { false }) \\
& \frac{\bar{e} \notin \mathcal{B} \quad(\sigma, t) \mid \vDash \operatorname{Guard}(w)}{(\sigma, e, \mathcal{B}, \mathcal{N}) \Longrightarrow(\sigma, \epsilon, \mathcal{B} \cup\{\bar{e}\}, \mathcal{N})} \\
& e=(t, w, \text { false }) \\
& \frac{\bar{e} \in \mathcal{N} \quad(\sigma, t) \mid \vDash \operatorname{Guard}(w)}{(\sigma, e, \mathcal{B}, \mathcal{N}) \Longrightarrow(\sigma, \epsilon, \mathcal{B}, \mathcal{N} \backslash\{\bar{e}\})} \\
& e=(t, w, \text { true }) \\
& \bar{e} \notin \mathcal{B} \quad(\sigma, t) \mid=\operatorname{Guard}(w) \\
& \langle\operatorname{Body}(w), t, \sigma\rangle \Downarrow \sigma^{\prime} \\
& \mathcal{N}_{1}=\operatorname{GetSignals}\left(w, \sigma^{\prime}, \mathcal{B}\right) \\
& \mathcal{N}_{2}=\operatorname{GetBroadcasts}\left(w, \sigma^{\prime}, \mathcal{B}\right) \\
& \overline{(\sigma, e, \mathcal{B}, \mathcal{N}) \Longrightarrow\left(\sigma^{\prime}, \epsilon, \mathcal{B}, \mathcal{N} \cup \mathcal{N}_{1} \cup \mathcal{N}_{2}\right)} \\
& e=(t, w, \text { true }) \\
& \bar{e}=\min (\mathcal{N}) \quad(\sigma, t) \mid=\operatorname{Guard}(w) \\
& \langle\operatorname{Body}(w), t, \sigma\rangle \Downarrow \sigma^{\prime} \\
& \mathcal{N}_{1}=\operatorname{GetSignals}\left(w, \sigma^{\prime}, \mathcal{B}\right) \\
& \mathcal{N}_{2}=\operatorname{GetBroadcasts}\left(w, \sigma^{\prime}, \mathcal{B}\right) \\
& \overline{(\sigma, e, \mathcal{B}, \mathcal{N}) \Longrightarrow\left(\sigma^{\prime}, \epsilon, \mathcal{B} \backslash\{\bar{e}\},\left(\mathcal{N} \cup \mathcal{N}_{1} \cup \mathcal{N}_{2}\right) \backslash\{\bar{e}\}\right)} \\
& \frac{(\sigma, e, \mathcal{B}, \mathcal{N}) \Longrightarrow\left(\sigma^{\prime}, \epsilon, \mathcal{B}^{\prime}, \mathcal{N}^{\prime}\right)}{(\sigma, e:: \tau, \mathcal{B}, \mathcal{N}) \Longrightarrow\left(\sigma^{\prime}, \tau, \mathcal{B}^{\prime}, \mathcal{N}^{\prime}\right)}
\end{aligned}
$$

Figure 5. Transition relation for explicit-signal monitor traces. Given an event $e=(t, w, b), \bar{e}$ denotes $(t, w)$.

$\mathrm{m} 2$ before finishing the execution of $\mathrm{m} 1$ (violates (b)). Finally, the following trace is also not syntactically well-formed:

$$
\left[\left(1, w_{11}, \text { false }\right),\left(2, w_{21}, \text { true }\right),\left(1, w_{11}, \text { true }\right),\left(1, w_{12}, \text { true }\right)\right]
$$

In particular, it violates requirement (c) since thread 2 exists the monitor without getting blocked or finishing the execution of $\mathrm{m} 2$. On the other hand, the following trace is syntactically well-formed:

$$
\begin{gathered}
\quad\left(1, w_{11}, \text { false }\right),\left(2, w_{21}, \text { true }\right),\left(2, w_{22}, \text { false }\right), \\
\left(1, w_{11}, \text { true }\right),\left(1, w_{12}, \text { true }\right),\left(2, w_{22}, \text { true }\right)
\end{gathered}
$$

In this trace, thread 1 attempts to execute the body of $w_{11}$ but is blocked (i.e., $x>0$ evaluates to false). Then, thread 2 executes the first waituntil statement in method $\mathrm{m} 2$, but gets blocked on the second one. After thread 2 executes $w_{21}$, $x>0$ becomes true, and thread 1 is able to finish executing method $\mathrm{m} 1$. Finally thread 2 finishes executing method $\mathrm{m} 2$.

Semantics. We now define the semantics of implicit-signal monitors in terms of the feasibility of well-formed monitor traces. Given a monitor $M$ and a monitor state $\sigma$, we say that a trace $\tau$ is feasible under $\sigma$ iff (a) it is syntactically well-formed and (b) $(\sigma, \tau, \emptyset, \emptyset) \longrightarrow^{*}\left(\sigma^{\prime}, \epsilon, \_,-\right)$where $\longrightarrow{ }^{*}$ denotes the reflexive transitive closure of the transition relation $\longrightarrow$ defined in Figure 4 .

Transition relations for implicit-signal monitors are described in Figure 4 using judgments of the form

$$
(\sigma, \tau, \mathcal{B}, \mathcal{N}) \longrightarrow\left(\sigma^{\prime}, \tau^{\prime}, \mathcal{B}^{\prime}, \mathcal{N}^{\prime}\right)
$$




$$
\begin{aligned}
\operatorname{Events}(\mathcal{B}, p) & =\{(t, w) \mid(t, w) \in \mathcal{B} \wedge \operatorname{Guard}(w)=p\} \\
\operatorname{GetSignals}(w, \sigma, \mathcal{B}) & =\left\{\left(t^{\prime}, w^{\prime}\right) \mid(p, c) \in \operatorname{Signals}(w) \wedge\left(t^{\prime}, w^{\prime}\right)=\min (\operatorname{Events}(\mathcal{B}, p)) \wedge\left(c=\checkmark \vee\left(\sigma, t^{\prime}\right) \mid=p\right)\right\} \\
\operatorname{GetBroadcasts}(w, \sigma, \mathcal{B}) & =\left\{\left(t^{\prime}, w^{\prime}\right) \mid(p, c) \in \operatorname{Broadcasts}(w) \wedge\left(t^{\prime}, w^{\prime}\right) \in \operatorname{Events}(\mathcal{B}, p) \wedge\left(c=\checkmark \vee\left(\sigma, t^{\prime}\right) \mid=p\right)\right\}
\end{aligned}
$$

Figure 6. Auxiliary functions used in Figure 5

where $\mathcal{B}$ and $\mathcal{N}$ describe blocked and notified threads respectively. In particular, $(t, w) \in \mathcal{B}$ indicates that thread $t$ is currently blocked on the predicate of $w$. In contrast, $(t, w) \in \mathcal{N}$ indicates that thread $t$ should be woken up to recheck the predicate of $w$. The meaning of the judgment $(\sigma, \tau, \mathcal{B}, \mathcal{N}) \longrightarrow\left(\sigma^{\prime}, \tau^{\prime}, \mathcal{B}^{\prime}, \mathcal{N}^{\prime}\right)$ is that executing the first event $e$ in $\tau$ under $\sigma, \mathcal{B}$, and $\mathcal{N}$ yields a new state $\sigma^{\prime}$ as well as a new set of blocked and notified threads $\mathcal{B}^{\prime}$ and $\mathcal{N}^{\prime}$ respectively. We now explain the transition relations from Figure 4 in more detail.

According to rules (1a) and (1b), an event $e$ of the form $(t, w, f a l s e)$ is only feasible when $(\sigma, t) \quad \forall \operatorname{Guard}(w)$ (i.e., the predicate of $w$ evaluates to false). If $\bar{e}=(t, w)$ was not previously in the blocked thread set $\mathcal{B}$, rule (1a) adds $\bar{e}$ to $\mathcal{B}$. If $\bar{e}$ was already in $\mathcal{B}$, then $e$ is only feasible if $\bar{e}$ was "notified" by the system (i.e., $\bar{e} \in \mathcal{N}$ in rule (1b)).

The next two rules (2a) and (2b) state that an event $e=$ $(t, w$, true $)$ is only feasible when $(\sigma, t) \mid=\operatorname{Guard}(w)$ (i.e., the predicate of $w$ evaluates to true). Both rules execute the body of $w$ to obtain a new monitor state $\sigma^{\prime}$. Now, since the execution of $w$ may cause the predicates of blocked threads to become true, $\mathcal{N}^{\prime}$ contains all $(t, w)$ pairs that were previously in $\mathcal{B}$ and whose predicates evaluate to true under $\sigma^{\prime}$.

\subsection{Target Language}

Our target language is very similar to the source language from Figure 3, except that the body of waituntil statements contain explicit signals. In particular, a wai tuntil construct in the target language looks as follows:

$$
\text { waituntil }(p)\left\{s ; \operatorname{signal}\left(S_{1}\right) ; \operatorname{broadcast}\left(S_{2}\right)\right\}
$$

Here, $S_{1}$ and $S_{2}$ are sets of pairs $(p, c)$ where $p$ is a predicate and $c \in\{?, \checkmark\}$. The informal semantics of signal and broadcast are as follows: If $(p, \checkmark)$ is in $S_{1}$, then the system will notify (i.e., wake up) a single thread blocked on predicate $p$. In contrast, if $(p, \checkmark) \in S_{2}$, then the system notifies all threads blocked on $p$. On the other hand, if $(p, ?)$ is in $S_{1}$ (resp. $S_{2}$ ), then $p$ will be evaluated at run-time, and, if $p$ evaluates to true, then one thread (resp. all threads) blocked on $p$ will be notified. Given a waituntil statement in the target language, we write $\operatorname{Signals}(w)$ to indicate $S_{1}$ and $\operatorname{Broadcasts}(w)$ to represent $S_{2}$.

We also describe the formal semantics of explicit-signal monitors in terms of monitor traces, where the definitions of trace, event, and well-formedness remain the same as in Section 3.2. However, the concept of feasibility is defined with respect to a different transition relation $\Longrightarrow$, shown in Figure 5. In particular, we say that an explicit-signal monitor trace $\tau$ is feasible if (a) it is syntactically well-formed, and (b) $(\sigma, \tau, \emptyset, \emptyset) \Longrightarrow^{*}\left(\sigma^{\prime}, \epsilon,,-,\right)$ where $\Longrightarrow^{*}$ denotes the reflexive transitive closure of the transition relation $\Longrightarrow$ from Figure 5 .

The transition relation $\Longrightarrow$ is defined similarly as $\longrightarrow$ except for events of the form $(t, w$, true $)$. In contrast to implicit signal monitors which wake up all threads whose predicates have become true, explicit-signal monitors decide which threads to notify based on Signals $(w)$ and Broadcasts $(w)$. In particular, rules (2a) and (2b) use auxiliary functions GetSignals and GetBroadcasts (defined in Figure 6) to decide which threads to add to the notification set $\mathcal{N}$. If $(p, c) \in \operatorname{Signals}(w)$, then we notify a single event $\left(t^{\prime}, w^{\prime}\right) \in \mathcal{B}$ such that the predicate of $w^{\prime}$ is $p$. If $c=$ ?, we additionally check that $p$ evaluates to true under $\sigma^{\prime}$ before adding $\left(t^{\prime}, w^{\prime}\right)$ to the notification set. The function GetBroadcasts is defined similarly except that it notifies all threads blocked on the specified predicate rather than a single one.

\subsection{Equivalence}

We are now ready to define what it means for an implicitsignal monitor $M$ from the source language and an explicitsignal monitor $M^{\prime}$ from the target language to be equivalent. Towards this goal, we first define a normal form for traces:

Definition 3.3. (Normalization) Let $\tau$ be an implicitsignal monitor trace. We say that $\tau$ is normalized with respect to monitor state $\sigma$ if we can derive $(\sigma, \tau, \emptyset, \emptyset) \longrightarrow^{*}\left(\sigma, \epsilon,{ }_{-},\right)$ without using rule (1b) from Figure 4 in the derivation.

Since rule (1b) corresponds to a spurious notification, a trace is normalized if threads are woken up only when their predicates evaluate to true. Observe that we can always find a normalized feasible trace for any feasible trace by changing the order in which threads are woken up. ${ }^{3}$

Definition 3.4. (Equivalence) Let $M, M^{\prime}$ be implicit- and explicit-signal monitors respectively. We say that $M$ and $M^{\prime}$ are semantically equivalent, written $M \sim M^{\prime}$, iff for all monitor states $\sigma$ and all well-formed traces $\tau$, the following two conditions are satisfied:

1. If $(\sigma, \tau, \emptyset, \emptyset) \Longrightarrow^{*}\left(\sigma^{\prime}, \epsilon,{ }_{-},-\right)$, then it is also the case that $(\sigma, \tau, \emptyset, \emptyset) \longrightarrow^{*}\left(\sigma^{\prime}, \epsilon,{ }_{-},-\right)$.

2. If $(\sigma, \tau, \emptyset, \emptyset) \longrightarrow^{*}\left(\sigma^{\prime}, \epsilon,{ }_{-}\right)$and $\tau$ is normalized with respect to $\sigma$, then $(\sigma, \tau, \emptyset, \emptyset) \Longrightarrow *\left(\sigma^{\prime}, \epsilon,{ }_{-},{ }_{-}\right)$.

Here, the first condition states that any feasible trace of the explicit-signal monitor $M^{\prime}$ must also be a feasible trace of its implicit version $M$. However, in general, we cannot expect

\footnotetext{
${ }^{3}$ Recall that our notion of feasibility does not require the sets $\mathcal{B}, \mathcal{N}$ to be empty. In particular, a trace $\tau$ is feasible under $\sigma$ if $(\sigma, \tau, \emptyset, \emptyset) \longrightarrow *$ $\left(\sigma^{\prime}, \epsilon, \mathcal{B}, \mathcal{N}\right)$ for any $\mathcal{B}$ and $\mathcal{N}$. Therefore, a notification that would have been eliminated by rule (1b) can just be ignored indefinitely, i.e., remain in the $\mathcal{N}$ set without affecting other transitions.
} 


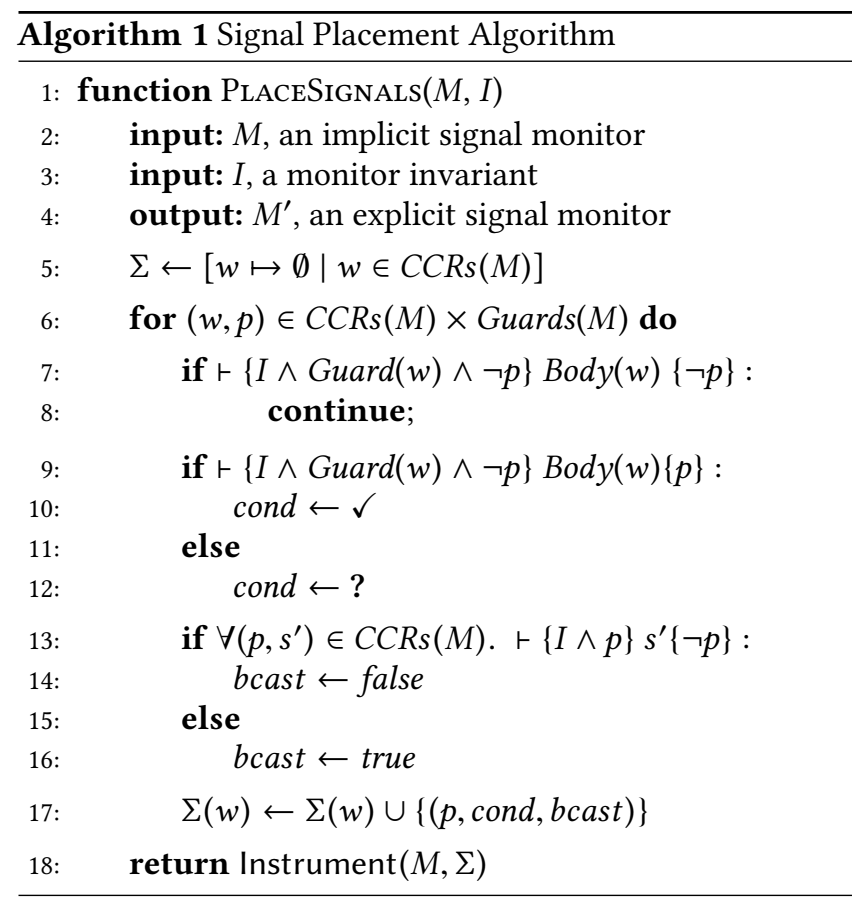

the converse of this statement to hold: Since the explicitsignal monitor may be more efficient than its implicit-signal counterpart, we cannot require that all feasible traces of $M$ to be also feasible in the explicit-monitor case. Thus, the second condition states that any normalized feasible trace of $M$ should also be feasible in $M^{\prime}$.

\section{Signal Placement Algorithm}

In this section, we describe our algorithm for automatically transforming an implicit-signal monitor $M$ in the source language to an explicit-signal monitor $M^{\prime}$ in the target language. Our algorithm ensures that $M$ and $M^{\prime}$ are equivalent in the sense of Definition 3.4 and also tries to minimize the number of spurious wake-ups and conditional signals in $M^{\prime}$. We start with a basic version of the algorithm and then describe extensions and improvements later in this section.

\subsection{Basic Algorithm}

Our basic signal placement algorithm is shown in Algorithm 1. The PlaceSignals algorithm takes as input an implicit-signal monitor $M$ as well as a monitor invariant $I$, which is an assertion that holds every time a thread enters and exits the monitor. Since automated inference of monitor invariants is described in the next section, we will assume that an oracle provides them for the time being. In this section, we further assume that guards used in waituntil statements do not contain thread-local variables. Given such an implicit-signal monitor $M$ and its invariant $I$, PLACESIGNALS returns an explicit-signal monitor $M^{\prime}$ such that $M \sim M^{\prime}$.

The algorithm maintains a mapping from each conditional critical region (CCR) (i.e., waituntil statement) $w$ in $M$ to a set of notifications that should be performed after executing $w$ and before exiting the monitor. The algorithm represents these notification as triples of the form ( $p$, cond, bcast), where $p$ is a predicate, cond $\in\{?, \checkmark\}$ indicates whether the notification is conditional or unconditional, and bcast is a boolean indicating whether it is necessary to notify all threads blocked on $p$ as opposed to a single one. Once the algorithm computes this mapping $\Sigma$, it instruments the original implicit-signal monitor $M$ as shown in Figure 7 to obtain an explicit-signal monitor $M^{\prime}$.

The key part of the PlaceSignals algorithm is the loop in lines 6-17. For each conditional critical region $w$ and predicate $p$ used in the monitor, the algorithm first decides whether $w$ may need to notify threads blocked on predicate $p$. This decision is made based on the provability of the following Hoare triple:

$$
\{I \wedge \operatorname{Guard}(w) \wedge \neg p\} \operatorname{Body}(w)\{\neg p\}
$$

Essentially, this triple says that executing the body of $w$ in a state in which $\neg p$ holds ensures that predicate $p$ continues to remain false. Hence, any thread $t$ blocked on $p$ will remain blocked after executing $w$, so there is no need to notify $t$. Observe that the precondition of the Hoare triples also assumes $I \wedge \operatorname{Guard}(w)$ because (a) $\operatorname{Guard}(w)$ is a prerequisite for executing the body of $w$ and, (b) by definition of monitor invariant, $I$ must hold before executing the body of any CCR.

Next, lines 9-12 determine whether the notification should be conditional or not. Recall that a conditional notification for predicate $p$ checks whether $p$ evaluates to true before waking up threads blocked on $p$. While conditional notifications prevent spurious wake-ups, it is desirable to avoid evaluating $p$ at run-time if $p$ is guaranteed to hold after executing $w$. Thus, line 9 checks the validity of the following Hoare triple:

$$
\{I \wedge \operatorname{Guard}(w) \wedge \neg p\} \operatorname{Body}(w)\{p\}
$$

In other words, assuming we execute $w$ in a state where a thread is blocked on $p$, the execution of $\operatorname{Bod} y(w)$ results in a state where $p$ is true. Thus, there is no need to evaluate $p$ at run time before signaling threads blocked on $p$.

The last part of Algorithm 1 (lines 13-16) determines whether we should notify all threads blocked on predicate $p$. Suppose there are $n$ threads $T=\left\{t_{1}, \ldots, t_{n}\right\}$ blocked on $p$, and suppose that an arbitrary thread $t_{i}$ gets unblocked. If executing $t_{i}$ is guaranteed to result in a state where predicate $p$ is false, then it is not necessary to notify any of the remaining threads $T \backslash\left\{t_{i}\right\}$. Thus, the algorithm checks the following Hoare triple for all CCRs $w^{\prime}$ with guard $p$ :

$$
\{I \wedge p\} \operatorname{Body}\left(w^{\prime}\right)\{\neg p\}
$$

If this Hoare triple holds for all CCRs with guard $p$, then it is safe to signal rather than broadcast.

Theorem 4.1. ${ }^{4}$ Let PlaceSignals $(M, I)=M^{\prime}$. If I is a correct monitor invariant and guards of CCRs in $M$ do not contain thread-local variables, then $M \sim M^{\prime}$.

\footnotetext{
${ }^{4}$ The proofs of all theorems are in the extended version of the paper [21].
} 


$$
\begin{gathered}
w=\text { waituntil }\left(p^{\prime}\right)\{s\} \\
S_{1}=\left\{(p, c) \mid(p, c, \text { false }) \in \sum(w)\right\} \\
S_{2}=\left\{(p, c) \mid(p, c, \text { true }) \in \sum(w)\right\} \\
s^{\prime}=\operatorname{signal}\left(S_{1}\right) ; \text { broadcast }\left(S_{2}\right) \\
\hline \sum \vdash w \leadsto \text { waituntil }\left(p^{\prime}\right)\left\{s ; s^{\prime}\right\} \\
\sum \vdash w_{1} \leadsto w_{1}^{\prime} \ldots \quad \sum \vdash w_{n} \leadsto w_{n}^{\prime} \\
\hline \sum \vdash w_{1} ; \ldots ; w_{n} \leadsto w_{1}^{\prime} ; \ldots ; w_{n}^{\prime}
\end{gathered}
$$

Figure 7. Performing instrumentation

\subsection{Handling Thread-Local Variables}

To simplify presentation, our algorithm from Section 4.1 assumes that guards of CCRs in the input monitor do not contain thread-local variables. However, if the input monitor $M$ does not satisfy this assumption, the explicit-signal monitor $M^{\prime}$ generated by Algorithm 1 may not be equivalent to $M$. We illustrate the problem using the following example:

Example 4.2. Consider the following monitor:

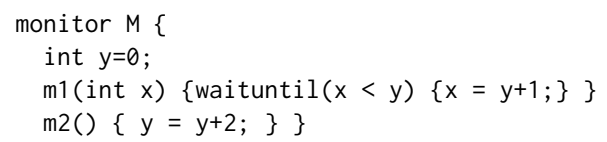

Suppose we have threads $t_{1}, t_{2}, t_{3}$, where $t_{1}, t_{2}$ are blocked in $\mathrm{m} 1$, and $t_{3}$ is executing $\mathrm{m} 2$, after which the value of $\mathrm{y}$ becomes 2. Further, suppose that the value of the threadlocal variable $\mathrm{x}$ is 0 for $t_{1}$ and 1 for $t_{2}$. Since the predicate $x<y$ has become true for both $t_{1}, t_{2}$ and executing $t_{1}$ does not change the value of the predicate in $t_{2}$ (and vice versa), $t_{3}$ should notify both threads. Thus, the explicit-signal monitor should use broadcast instead of signal.

However, recall that Algorithm 1 determines whether $\mathrm{m} 2$ should broadcast or signal by checking the validity of $\{x<$ $y\} x=y+1\{x \geq y\}$. Since this Hoare triple is valid, we would erroneously conclude that it is safe for $\mathrm{m} 2$ to notify a single thread instead of all threads.

As illustrated by this example, Algorithm 1 is unsound when guards contain thread-local variables. To remedy this situation, we need to rename thread-local variables when checking validity. In particular, recall that PlaCESignals checks the validity of Hoare triples of the form $\left\{P_{1} \wedge P_{2}\right\} S\{Q\}$ where $P_{1}$ is an assumption about the currently running thread, whereas $P_{2}$ and $Q$ are assumptions/assertions about some other thread. Since $S$ and $P_{1}$ may refer to thread-local variables that are also used in $P_{2}$ and $Q$, we need to rename thread-local variables and check the validity of the following modified Hoare triple:

$$
\left\{P_{1} \wedge P_{2}\left[V^{\prime} / V\right]\right\} S\left\{Q\left[V^{\prime} / V\right]\right\}
$$

where $V=\operatorname{Locals}\left(P_{2}\right) \cup \operatorname{Locals}(Q)$ and $V^{\prime}$ denotes a fresh set of variables not used elsewhere in $P_{1}, P_{2}, S$, and $Q .^{5}$

\footnotetext{
${ }^{5}$ Another subtlety with local variables is how to signal conditionally. We discuss evaluation of predicates with local variables in Section 6.
}

\subsection{Improvement over the Basic Algorithm}

In this section, we consider an improvement over Algorithm 1 that aims to further reduce the number of broadcasts in the synthesized explicit-signal monitor. Recall that Algorithm 1 determines whether a CCR should notify one vs. all threads blocked on predicate $p$ by checking the validity of the following Hoare triple for all CCRs $w$ with guard $p$ :

$$
\{I \wedge p\} \operatorname{Body}(w)\{\neg p\}
$$

In some cases, it is possible to further strengthen the precondition of this Hoare triple. In particular, suppose that the signaling CCR is $w^{\prime}$ with body $s^{\prime}$ and guard $p^{\prime}$ and suppose that $\phi$ is guaranteed to hold after executing $s^{\prime}$. In general, we cannot assume $\phi$ in the pre-condition of Equation 1 because other threads may have invalidated $\phi$ before the notified thread has a chance to execute. However, if $s$ commutes with the body of every other CCR in the monitor, then the monitor state after executing $s$ for any interleaving is equivalent to one in which we execute $s$ immediately after $s^{\prime}$. In this case, we can safely assume that $s$ executes immediately after $s^{\prime}$ since the resulting states are equivalent. This insight allows us to strengthen the precondition of Equation 1 by using the post-condition $\phi$ of the signaling thread.

To make this discussion more precise, let us define a predicate $\operatorname{Comm}(w, M)$ as follows:

$$
\begin{gathered}
\operatorname{Comm}(w, M) \Leftrightarrow\left(\forall w^{\prime} \in \operatorname{CCRs}(M) \backslash\{w\} .\right. \\
\left.\operatorname{Body}\left(w^{\prime}\right) ; \operatorname{Body}(w) \equiv \operatorname{Body}(w) ; \operatorname{Body}\left(w^{\prime}\right)\right)
\end{gathered}
$$

Essentially, this predicate is true if the body of $w$ commutes with every other CCR in the monitor. Now, using this definition, we can state a weaker sufficient condition for CCR $w$ to notify one -rather than all- threads blocked on predicate $p$. In particular, we can change the condition at line 13 of Algorithm 1 to the following weaker one:

$$
\begin{gathered}
\forall w^{\prime}=\left(p, s^{\prime}\right) \in \operatorname{CCRs}(M) .\left(\vdash\{I \wedge p\} s^{\prime}\{\neg p\} \vee\right. \\
\left.\left(\operatorname{Comm}\left(w^{\prime}, M\right) \wedge \vdash\{I \wedge \operatorname{Guard}(w) \wedge \neg p\} \operatorname{Body}(w) ; s^{\prime}\{\neg p\}\right)\right)
\end{gathered}
$$

The first line of Equation 2 corresponds to the same check we perform at line 13 in Algorithm 1 to determine whether it is safe to signal rather than broadcast. However, if this condition does not hold, we may still be able to prove that broadcasting is unnecessary as long as $s^{\prime}$ commutes with every other CCR in the monitor and we can prove that $p$ is falsified after executing $\operatorname{Body}(w) ; s^{\prime}$.

The correctness of Equation 2 follows from the following theorem (and the proof of Theorem 4.1):

Theorem 4.3. Let $\tau=\tau_{0}$ e be a monitor trace and let $\tau^{\prime}=e \tau_{0}$ where $e=(t, w, b)$. If $(\sigma, \tau, \mathcal{B}, \mathcal{N}) \longrightarrow^{*}\left(\sigma^{\prime}, \epsilon, \mathcal{B}^{\prime}, \mathcal{N}^{\prime}\right)$ and $\operatorname{Comm}(w, M)$, then we have $\left(\sigma, \tau^{\prime}, \mathcal{B}, \mathcal{N}\right) \longrightarrow^{*}\left(\sigma^{\prime}, \epsilon, \mathcal{B}^{\prime}, \mathcal{N}^{\prime}\right)$.

Remark. Our discussion in this section assumes nonpreemptive signal semantics [3] where a signaled thread is not guaranteed to consume the signal immediately. However, if we assume preemptive signal semantics, we can perform this optimization more liberally by only checking whether 


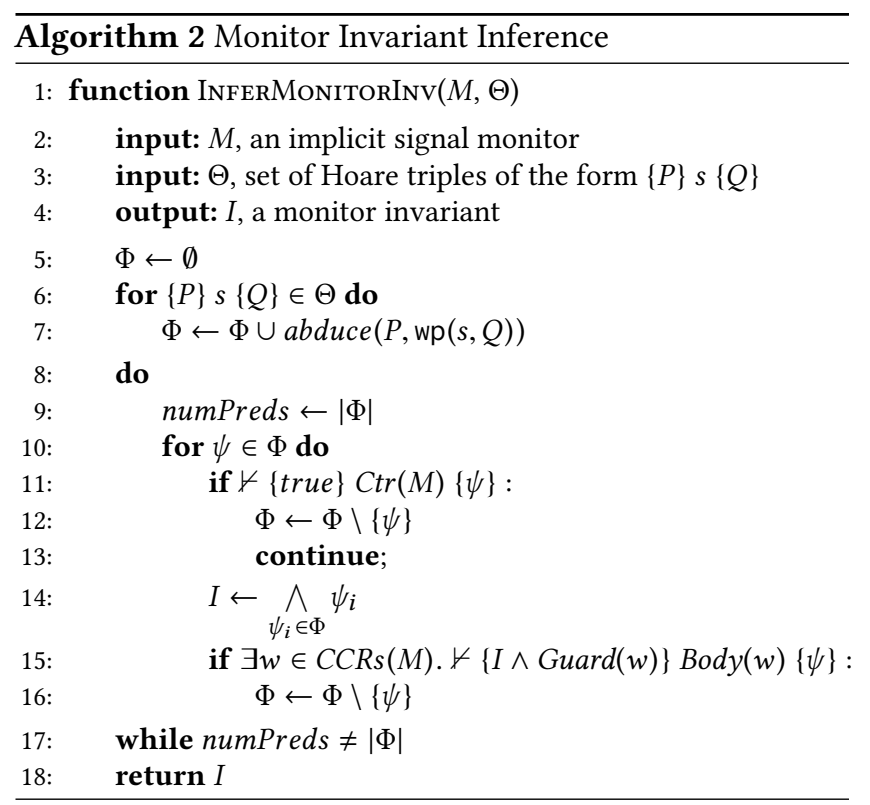

predicate $p$ is invalidated by the sequential composition of the segment that produces the signal and $\operatorname{Bod} y(w)$.

\section{Inference of Monitor Invariants}

Our signal placement algorithm from Section 4 relies on a monitor invariant $I$ that holds at the entry and exit of every CCR. In this section, we describe our method for automatically inferring useful monitor invariants.

Our inference algorithm is property-directed in that it only infers invariants that are useful for proving the Hoare triples generated by the signal placement algorithm. Specifically, our inference engine uses abductive reasoning [16] to automatically infer predicates that are useful for proving a given set of Hoare triples. Given a universe of predicates $\Phi$ generated using abduction, it then infers the strongest conjunctive monitor invariant over predicates in $\Phi$. Therefore, our invariant inference engine can be viewed as marrying the power of abductive reasoning with predicate abstraction [22, 38]. The advantage of this approach is two-fold: First, rather than relying on a hard-coded universe of predicate templates, our algorithm infers useful predicates automatically using abduction. Second, because the predicates inferred using abduction can involve disjunctions, the monitor invariants synthesized by our algorithm are not restricted to pure conjunctions.

With this intution in mind, we now explain our INFERMonitorInv procedure from Algorithm 2 in more detail. This procedure takes two inputs, namely, an implicit-signal monitor $M$ and a set $\Theta$ of Hoare triples of the form $\{P\} s\{Q\}$. Note that $\Theta$ simply corresponds to the set of Hoare triples generated by Algorithm 1, but with I set to true. The return value of INFERMONITORINV is a formula $I$ representing a valid monitor invariant of $M$.

Conceptually, the INFERMONITORINv procedure operates in two phases. The first phase (lines 5-7) generates a universe
$\Phi$ of candidate predicates, and the second phase (lines 817) performs fixed-point computation to infer the strongest conjunctive monitor invariant $I$ over predicates in $\Phi$.

In the first phase of the algorithm, we iterate over all Hoare triples $\{P\} s\{Q\}$ in $\Theta$ and look for a strengthening $\psi$ of the precondition such that the Hoare triple $\{P \wedge \psi\} s\{Q\}$ becomes valid. Because the correctness of the Hoare triple $\{P\} s\{Q\}$ boils down to checking the validity of the formula $P \Rightarrow$ wp $(s, Q)$, we can find a suitable strengthening of $P$ by solving the following abductive reasoning problem:

Find $\psi$ such that :
(1) $P \wedge \psi \mid=w p(s, Q)$
(2) $P \wedge \psi \nLeftarrow$ false

Here condition (1) states that $\{P \wedge \psi\} s\{Q\}$ is a valid Hoare triple, and (2) states that the speculated invariant $\psi$ is consistent with precondition $P$. Since abductive reasoning is a well-studied problem, we use the abduce procedure described in prior work [16] to automatically infer candidate strengthenings $\psi$. Also, note that a call to abduce at line 7 may yield multiple predicates $\psi_{1}, \ldots, \psi_{n}$, all of which constitute valid solutions for Equation 3.

Since the predicates $\Phi$ generated using abduction in lines 5-7 are merely candidate invariants, the next phase of the algorithm performs a fixed-point computation in which we drop every $\psi \in \Phi$ that is not a monitor invariant. Specifically, for each predicate $\psi$ in $\Phi$, we check whether (a) it holds initially (lines 11-13) and (b) whether it is preserved by each CCR in the monitor (lines 15-16). To determine whether $\psi$ holds initially, we check the validity of the Hoare triple $\{\operatorname{true}\} \operatorname{Ctr}(M)\{\psi\}$, where $C \operatorname{tr}(M)$ denotes the constructor of $M .^{6}$ If this Hoare triple is not valid, we simply drop $\psi$ from set $\Phi$. Next, to determine whether $\psi$ is preserved by CCR $w$, we check the validity of the Hoare triple $\{I \wedge \operatorname{Guard}(w)\} \operatorname{Body}(w)\{\psi\}$, where $I$ denotes the conjunction of all predicates in $\Phi$. If this triple is invalid for any CCR in $M$, we again drop $\psi$ from the set $\Phi$. We then repeate this process until $I$ satisfies both the initiation and consecution requirements. It is easy to see that formula $I$ returned by INFERMONITORINV constitutes a valid monitor invariant.

\section{Implementation}

We have implemented our proposed method in a tool called Expresso. Our implementation leverages the Soot program analysis infrastructure [47] and invokes the Z3 SMT solver [12] for checking logical validity. In what follows, we discuss some important design choices that are not addressed in previous sections.

Generating Java code. While the target language (IR) presented in Section 3.3 is convenient for describing our transformation, it does not yield valid Java code. Our implementation converts programs in this IR to valid Java code in the following manner. First, we associate a condition variable with the guard of every waituntil statement. Now, given a

\footnotetext{
${ }^{6}$ For simplicity, we assume a single constructor; if there are multiple ones,
} this triple needs to be checked for all constructors. 
waituntil statement $w$ with associated guard $p$, body $s$, and condition variable $c$, we then generate the following $\operatorname{code}^{7}$ :

$$
\text { while(!p) }\{c \text {.await (); }\} ;
$$

Furthermore, for each $\left(p_{i}, ?\right) \in \operatorname{Signals}(w)$, we generate the code if $\left(p_{i}\right) c_{i}$.signal () , and for $\left(p_{i}, \checkmark\right) \in \operatorname{Signals}(w)$, we emit $c_{i}$.signal(). For each $\left(p_{i},{ }_{-}\right) \in \operatorname{Broadcasts}(w)$, we generate the same code where signal is replaced with signalAll.

Instrumentation for predicates with local variables. To support conditional signaling for predicates with local variables, ExPRESSo augments the monitor code with a data structure that tracks the values of local variables for any thread that is blocked on a predicate $p$. The code generated by ExPREsso then uses this data structure to check whether $p$ actually evaluates to true at program points that require conditional signaling for $p$.

Lazy broadcasts. EXPRESSO provides an option for performing broadcasts lazily. Consider a waituntil statement $w$ such that $\left(p,{ }_{-}\right) \in$ Broadcasts $(w)$. Rather than emitting the code c.signalAll() after the body of $w$, "lazy broadcast" notifies a single thread $t$ blocked on $p$ and ensures that $t$ notifies all other threads by adding the instrumentation if $(p)$ c.signal() after every waituntil statement with guard $p$. In our implementation, we enable this option by default to minimize context switches.

Discharging Hoare triples. ExPRESSO discharges any Hoare triple $\{P\} s\{Q\}$ by computing the weakest precondition of $Q$ with respect to $s$ and perfoming a validity check. Since $s$ can contain pointers, ExpREsso uses the points-to information provided by Doop [6] to produce a whole-program model of the heap. In particular, given a store statement $v \cdot f=e$, Expresso generates additional statements of the form if $\left(v=x_{i}\right) x_{i} . f=e$ where $x_{i}$ is a potential alias of $v$.

\section{Evaluation}

We evaluate ExPREsso by performing experiments that are designed to answer the following research questions:

- How does the code generated by Expresso compare against hand-written explicit-signal code?

- How does our solution compare against run-time systems that provide support for implicit signals?

- How long does Expresso take to generate code?

Benchmarks. The benchmarks used in our evaluation come from two different sources, namely all AutoSynch benchmarks from [31] and monitors collected from popular opensource projects from Github. We collected the Github benchmarks by writing a crawler that identifies potential monitors in Java programs using keywords such as wait, signal, notify etc. We then manually inspected these results in decreasing order of Github ranking (a mix of stars and forks) and identified self-contained modules (i.e., monitors) that encapsulate shared state. This process requires manual effort

\footnotetext{
${ }^{7}$ Note that our implementation uses the ReentrantLock class.
}

because we need to isolate the monitor code and insert it in a stress-testing harness.

Performance evaluation methodology. We evaluate performance using the same methodology used for evaluating AutoSynch in [31]. Specifically, we use saturation tests [8] in which threads only access the monitor and perform no extra work outside of the monitor. This set-up allows us to stress-test the monitor code and meaningfully compare our solution with run-time solutions and near-optimal handwritten code (from the original AutoSynch benchmarks or from the GitHub project).

We perform measurements using the JMH framework [45], which is a benchmarking tool for rigorous measurement in JVM-based languages. All measurements are conducted on a 16-way (8 core x 2 SMT) Intel Xeon CPU E5-2640 v3 $2.60 \mathrm{GHz}$ with 132 GB of memory using JDK 1.8.0_101-b13.

Performance results. The results of our performance evaluation are presented in Figures 8 and 9. Specifically, Figure 8 shows the results for the AutoSynch benchmarks, augmented with the readers-writers example of Section 2. Figure 9 presents results for monitors found in popular GitHub projects. Each graph plots the average time (in milliseconds) per monitor operation (e.g., enterReader) against the number of threads.

In virtually all cases, the performance of ExPRESSOgenerated code is very close to hand-written explicit-signal code. The only significant differences are in the "H2O Barrier" benchmark under low concurrency and "Dining Philosophers" under high concurrency. In the latter, the explicit signalling code has knowledge of the problem structure itself, so it avoids all wakeups that do not lead to progress.

Comparing to AutoSynch, ExPREsso outperforms it by $1.56 \mathrm{x}$ on average over all benchmarks. ExPRESSO significantly outperforms AutoSynch for about half the benchmarks of Figure 8 , which are chosen or written by the AutoSynch authors themselves. On a few occasions, AutoSynch slightly outperforms ExPRESSO-generated code. As we discuss in Section 8, AutoSynch offers dynamic structures for quick inequality comparisons between shared variables and local values (which are captured as constants while the thread is waiting). This custom optimization can also be added to ExPREsso but the emphasis of our work has been on statically eliminating unnecessary signalling, rather than minimizing the overhead of dynamic checks.

The monitors found in GitHub projects (Figure 9) are more representative of synchronization patterns in-the-wild. ExPRESSO performs very well on these benchmarks, matching hand-optimized code and significantly outperforming AutoSynch: by $1.62 \mathrm{x}$ on average, and up to $2.5 \mathrm{x}$ on a highconcurrency setting with 128 threads.

Upon closer inspection of these benchmarks, we observe that the symbolic reasoning needed to achieve the results from Figure 9 is far from trivial. As a simple example, "ConcurrencyThrottle" from the Spring framework has a waiting 

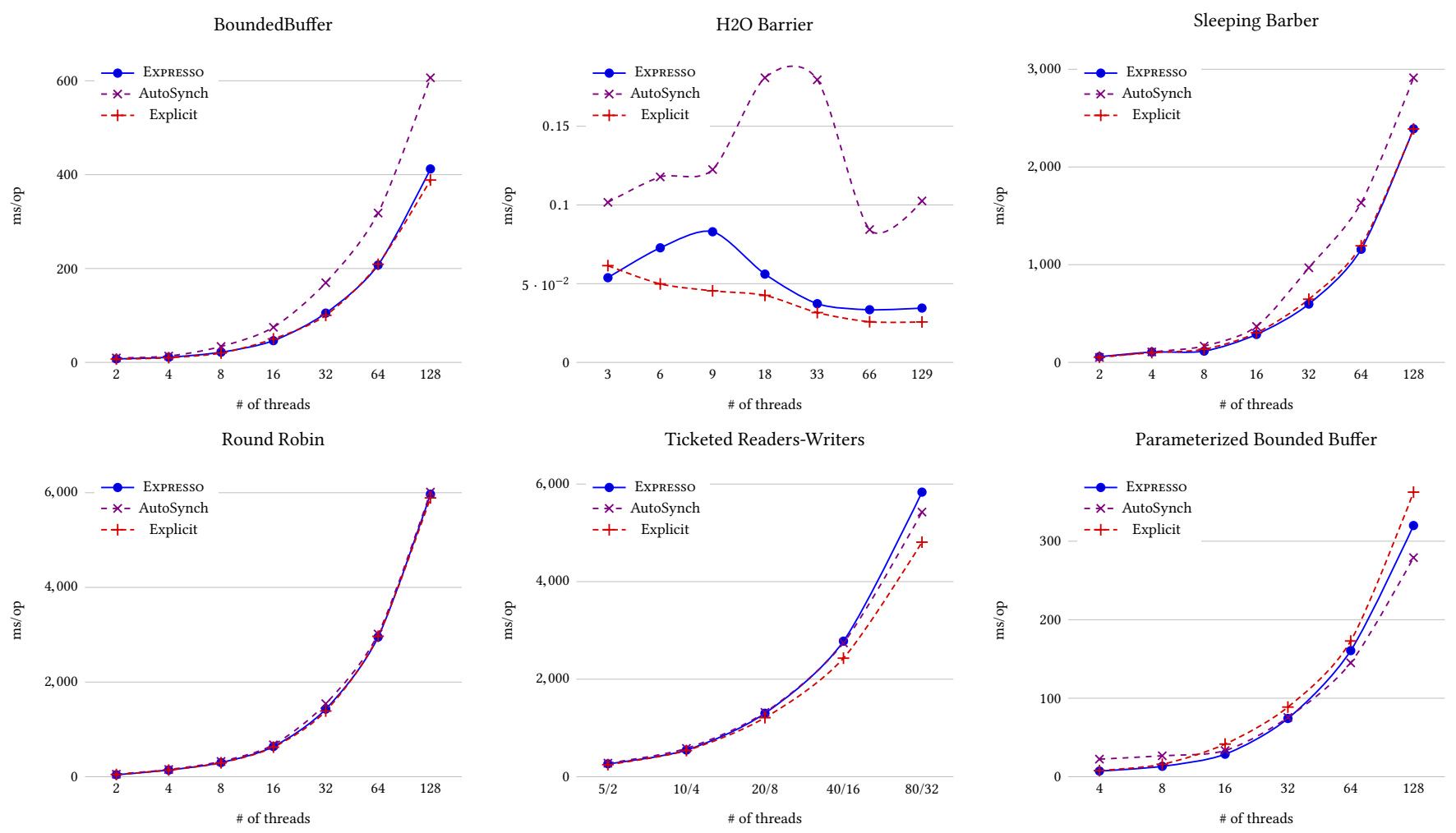

Dining Philosophers
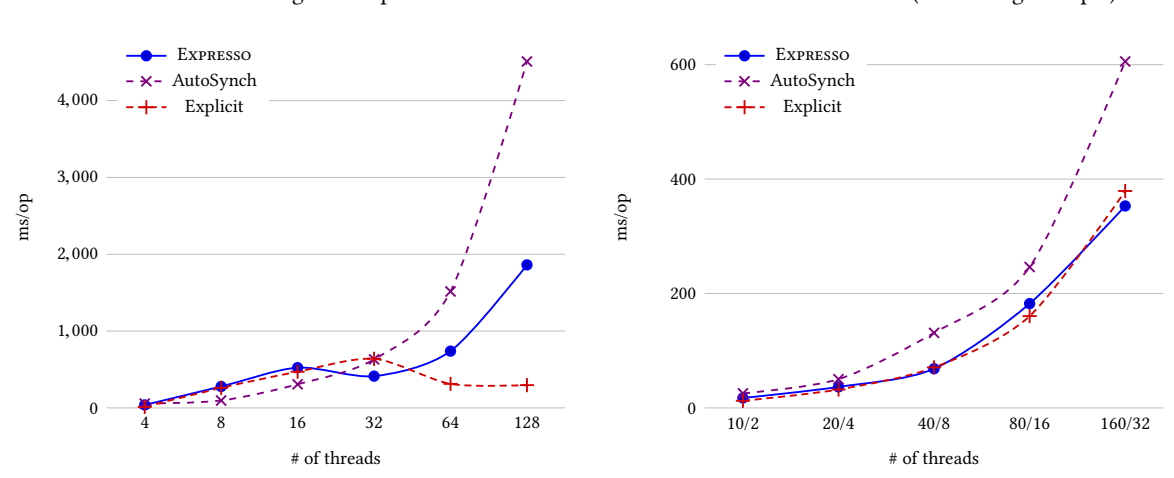

Figure 8. Performance over AutoSynch benchmarks and readers-writers example.

condition threadCount $<$ threadLimit triggered by the statement threadCount-- in the monitor exit operation. In order to avoid broadcasts, ExPRESSO needs to infer a monitor invariant that allows it to establish that whenever a thread enters the monitor, the waiting condition has to become true again due to a threadCount++ operation. Because these increment and decrement operations are distant, symbolic reasoning has to model the semantics of all intervening program statements and establish that the operations commute. This kind of reasoning is necessary for ExPREsso to achieve the performance results from Figure 9 in all benchmarks. Furthermore, the inferred monitor invariants are often intricate-for instance, the "AsyncDispatch" invariant (shown in the extended version of the paper [21]) from the Gradle codebase has 22 sub-terms (12 equality/inequality comparisons and arithmetic operations and 10 logical connectives).

To summarize, these results demonstrate the plausibility of a practical and efficient implementation for implicit-signal monitors. In particular, the code generated by ExPRESSO is comparable to hand-written code even for saturation tests that stress-test the monitor. Furthermore, ExPREsso's implicit-signal monitor implementation consistently outperforms AutoSynch on monitors extacted from real-world codebases such as Spring framework, Gradle, ExoPlayer, green$\mathrm{DAO}$, etc.

Analysis time. Table 1 shows the time that ExPresso takes to synthesize the explicit-signal code from its corresponding implicit-signal version for each benchmark. In most cases, 

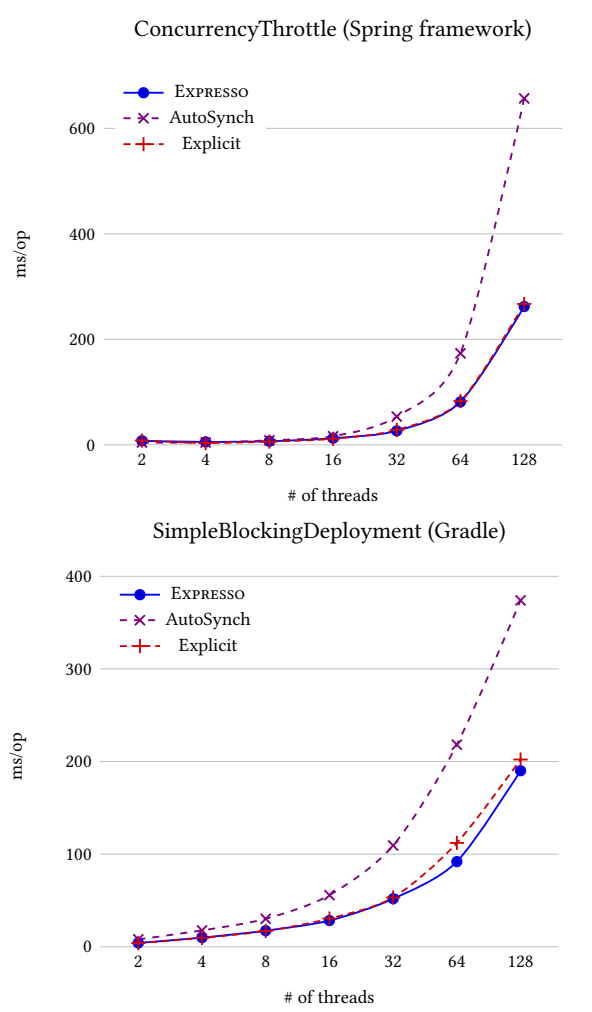

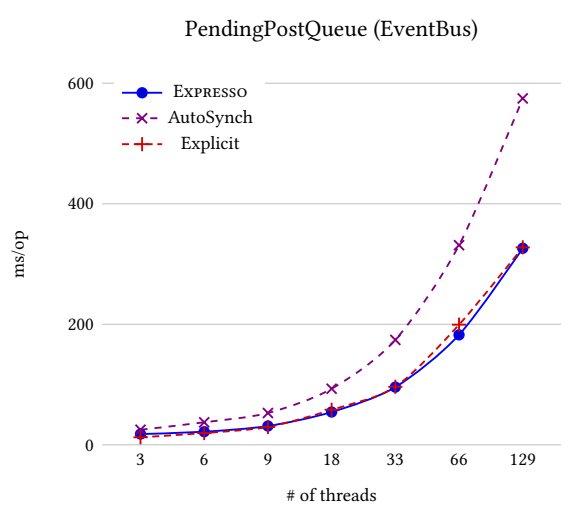

SimpleDecoder (ExoPlayer)

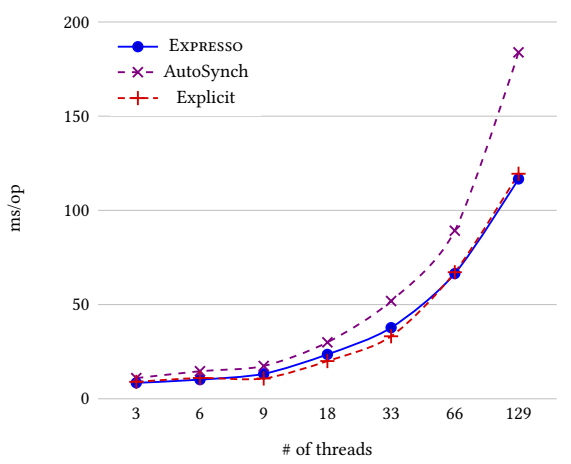

AsyncDispatch (Gradle)

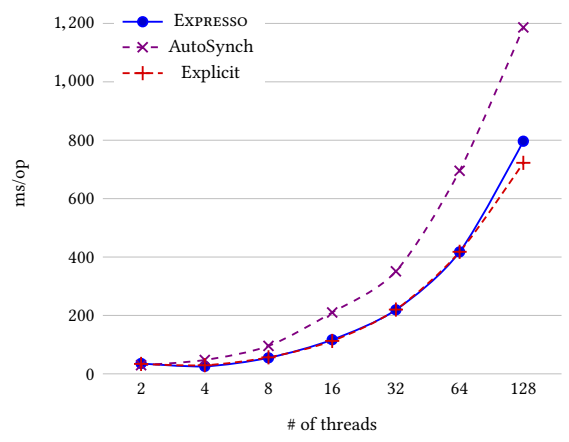

AsyncOperationExecutor (greenDAO)

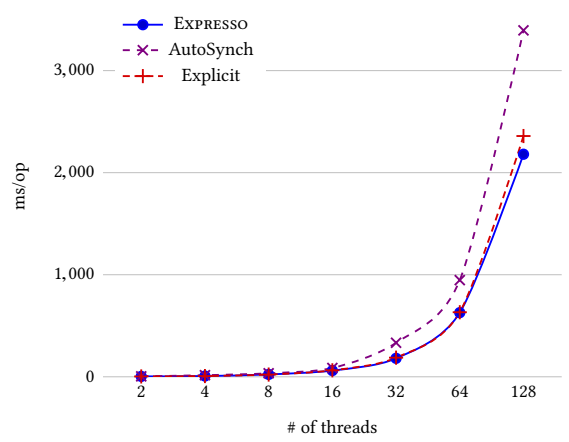

Figure 9. Performance over monitor code in popular GitHub projects.

the symbolic reasoning time is in the order of a few seconds. The only exception is the largest benchmark, AsyncDispatch, whose compilation takes 28.3 seconds. This example takes longer to analyze because some of the predicates depend on Java library operations that ExpREsso also needs to analyze. Overall, these results demonstrate that ExPREsso is practical and that it generates code whose performance is comparable to hand-written code in virtually all cases.

Generated code. We also assessed the quality of the code generated by ExPREsso by manually inspecting the synthesized explicit-signal monitors. For most benchmarks obtained from Github projects, we found that the code generated by ExPRESSO is very similar to hand-written code. In the case of the AutoSynch benchmarks, however, we found some examples (e.g., Dining Philosophers) where the Expressogenerated code differs significantly from hand-written code. For these benchmarks, manually-written code leverages dynamic data structures to achieve optimal signaling, whereas ExPRESSO uses the fixed strategy described in Section 6 for handling predicates with local variables.

\section{Related Work}

Our implicit-signal monitors have several relatives in the literature. We discuss representative past work, in loose thematic groupings of decreasing affinity with our work.

Language designs and run-time support for concurrency. There is a very rich literature on language support for concurrency, dating back to the early 1960s [3]. Dijkstra originally proposed the concept of semaphores to provide a friendlier and more efficient programming abstraction than the busy-wait design [15]. In later work, Hoare proposed the concept of conditional critical regions (CCR) [28], which overcome some of the difficulties associated with semaphores by providing a more structured notation for specifying synchronization. In particular, every shared variable in a CCR must belong to a resource, and variables in a resource can only be accessed within so-called region statements of the form

\begin{tabular}{lc}
\hline Benchmark & Time (sec.) \\
\hline BoundedBuffer & 2.5 \\
H2OBarrier & 2.3 \\
Sleeping Barber & 1.6 \\
Round Robin & 1.2 \\
Ticketed Readers-Writers & 3.8 \\
Param. Bounded Buffer & 2.5 \\
Dining Philosophers & 5.4 \\
Readers-Writers & 1.5 \\
\hline ConcurrencyThrottle & 1.0 \\
PendingPostQueue & 0.5 \\
AsyncDispatch & 28.3 \\
SimpleBlockingDeployment & 0.4 \\
SimpleDecoder & 10.7 \\
AsyncOperationExecutor & 2.1 \\
\hline
\end{tabular}

Table 1. Compilation time for benchmarks. 
region $r$ when $\mathrm{B} \rightarrow \mathrm{S}$, where $B$ is a guard and $S$ is a statement. Concurrently to the introduction of CCRs, Dijkstra proposed the notion of monitors, which provide more structure than conditional critical regions and can be implemented as efficiently as semaphores [14]. To this day, monitors remain a popular concurrent programming paradigm, and the "monitor pattern" is widely used in many programming languages, including Java and $\mathrm{C}++$.

Even though early proposals for monitors advocate an implicit signaling mechanism [3, 4], most modern monitor implementations use explicit signaling due to performance considerations. More recent work in this area aim to popularize implicit signal monitors by providing a more efficient implementation [8, 31]. For example, the recent AutoSynch work [31] attempts to improve the efficiency of automatic signaling through a combination of efficient dynamic indexing and simple static analysis. AutoSynch offers sophisticated handling of local state in a thread. If threads wait on conditions based on standard equality/inequality patterns over local variables, the system dynamically snapshots the values of local variables and treats them as run-time constants-the variable values cannot have changed while the thread is waiting. As a result, the predicates have a standard structure of comparisons with constants. The efficient notification algorithm then works much like database indexing: it computes, given which shared values changed, what waiting predicates could possibly have been affected. Our approach is distinguished by its use of reasoning techniques for statically inferring when a condition must,may-not, or may have become true. In order to do so, our technique needs to take into consideration several issues that are not addressed by previous work, e.g., handling of memory aliases, interprocedural reasoning, etc. As shown in the evaluation, our method significantly outperforms the AutoSynch solution on many benchmarks and is comparable to hand-written code for most examples.

Transactional memoory. CCRs have been recently reified in several language designs, such as that of Harris and Frasier [25]. Such designs can be viewed as special cases of transactional memory (TM) [26], which has attracted enormous attention in the literature, as an alternative of lockbased synchronization [39].

Our implicit-signal monitors are both less and more ambitious than transactional memory techniques, in different respects. Monitors do not attempt to automate-away lockbased synchronization: we explicitly require the programmer to declare different resources together with their synchronization policy. Concretely, each monitor can be thought of as a single lock, whereas transactional memory techniques do away with distinctions between locks in favor of a single atomic construct. The same essential feature is kept in Harris and Frasier's conditional critical regions [25]: although atomic statements can have a condition associated with them, their code blocks are guaranteed to execute with atomic semantics, relative to any other atomic block, under whichever condition. Therefore, implicit-signal monitors are inherently lower-level than TM approaches, requiring more care on behalf of the programmer, but also imposing no overhead for maintaining atomic semantics. At the same time, our implicit-signal monitors are more ambitious on the implementation side. Static reasoning over abstract conditions results in the removal of extraneous signaling overheads, enabling high-performance execution.

Automation of synchronization. Static analysis has been applied to the optimization of synchronization primitives in the past, mostly in the context of auto-locking or lockinference techniques [11, 20, 27, 35, 42]. The language model these techniques implement is much closer to transactional memory than implicit-signal monitors: the analysis attempts to infer which locks protect which shared data, as well as which data are not thread-shared. Furthermore, these static analyses typically produce a whole-program model of shared data and do not reason over symbolic conditions, as in our approach. Techniques that infer synchronization given specifications and abstractions $[10,19,48]$ often use similar reasoning techniques as our approach (e.g., SMT solvers), but start from much more abstract input and place an emphasis on correctness, not on approaching hand-written code performance. Similar comments apply to approaches that synthesize synchronization actions, given abstract models of program behavior, using control theory techniques [36, 49].

Program analysis for concurrency. A major application of static analysis in the domain of concurrency has been in guaranteeing safety or finding bugs. There are techniques for ensuring safe programming using advanced typing [5], analyses for static race detection [44], approaches to concurrency bug fixing [32], tools that flag suspicious concurrency patterns $[30,50]$, and much more. Additionally, dynamic techniques for concurrency bug detection [1, 13, 34, 41, 46] often benefit from symbolic reasoning, especially in approaches inspired by model checking or symbolic execution techniques $[33,37,43]$. Such past work is only superficially related to ours, since the aims, programming abstractions, and analysis techniques used are quite different.

Abductive reasoning in program analysis. Our proposed approach uses abductive reasoning for automatically inferring monitor invariants. The use of abduction in program analysis is quite common, especially in the context of modular analysis [2, 9, 18], loop invariant generation [17], and specification inference [2, 51]. However, our use of abductive reasoning differs from prior work in that we use abduction to generate candidate predicates over which we synthesize monitor invariants using predicate abstraction.

\section{Limitations}

While this paper takes a first step towards synthesizing efficient explicit-signal implementations of implicit-signal monitors, it makes several assumptions that allow the proposed approach to be practical. First, we assume that predicates 
appearing in waituntil statements are expressible in some first-order theory (e.g., linear arithmetic). Hence, if the predicate corresponds to the result of a complicated function containing loops or recursion, our approach must be conservative and potentially broadcast at the end of every CCR. However, we believe that such cases are are in practice, allowing EXPRESSo to generate efficient code for monitors taken from real-world applications.

Second, our approach assumes that monitors do not contain nested waituntil statements and that calls that occur inside the body of a monitor method are closed calls [3], meaning that they do not release access to the monitor. We believe that the latter assumption is quite realistic, since most modern implementations of monitor-like constructs also assume closed-call semantics. On the other hand, handling nested waituntil statements is an interesting direction for future work. In particular, we plan to investigate how to leverage more sophisticated forms of symbolic reasoning to analyze implicit-signal monitors with nested waituntil statements.

\section{Conclusion}

We have presented a new technique for automatic signalling in concurrent programs. Our method statically analyzes an implicit-signal monitor implementation to determine where signaling is necessary and automatically generates its corresponding explicit-signal version. Our method employs symbolic reasoning to avoid unnecessary context switches and run-time evaluation of predicates. We have implemented the proposed algorithm in a tool called ExPRESSO and evaluate it on 14 benchmarks. Our evaluation shows that the code generated by EXPRESSO is very similar to hand-written code and that it significantly outperforms a state-of-the-art run-time solution in most cases.

\section{Acknowledgments}

We would like to thank Doug Lea, the anonymous reviewers, and the members of the UToPiA group for their insightful feedback. The first three authors of this paper are supported in part by NSF Award \#1712060, NSF Award \#1453386, AFRL Award \#8750-15-2-0096, and the last author is supported by ERC grant 307334 (SPADE), a Facebook Research and Academic Relations Program award, and an Oracle Labs collaborative research grant.

\section{References}

[1] Rahul Agarwal and Scott D. Stoller. 2006. Run-time Detection of Potential Deadlocks for Programs with Locks, Semaphores, and Condition Variables. In Proceedings of the 2006 Workshop on Parallel and Distributed Systems: Testing and Debugging (PADTAD '06). ACM, New York, NY, USA, 51-60. https://doi.org/10.1145/1147403.1147413

[2] Aws Albarghouthi, Isil Dillig, and Arie Gurfinkel. 2016. Maximal specification synthesis. In ACM SIGPLAN Notices, Vol. 51. ACM, 789801.

[3] Gregory R. Andrews. 1991. Concurrent Programming: Principles and Practice. Benjamin-Cummings Publishing Co., Inc., Redwood City, CA, USA.
[4] Russell Atkinson and Carl Hewitt. [n. d.]. Synchronization in actor systems. In POPL '77: Proc. 4th symposium on Principles of Programming Languages. ACM Press, 267-280. https://doi.org/10.1145/512950.512975

[5] Chandrasekhar Boyapati, Robert Lee, and Martin Rinard. 2002. Ownership types for safe programming: Preventing data races and deadlocks. In ACM Sigplan Notices, Vol. 37. ACM, 211-230.

[6] Martin Bravenboer and Yannis Smaragdakis. 2009. Strictly Declarative Specification of Sophisticated Points-to Analyses. In ACM Symposium on Object Oriented Programming: Systems, Languages, and Applications (OOPSLA). ACM Press, 243-262.

[7] Peter A Buhr, Michel Fortier, and Michael H Coffin. 1995. Monitor classification. ACM Computing Surveys (CSUR) 27, 1 (1995), 63-107.

[8] Peter A Buhr and Ashif S Harji. 2005. Implicit-signal monitors. ACM Transactions on Programming Languages and Systems (TOPLAS) 27, 6 (2005), 1270-1343.

[9] Cristiano Calcagno, Dino Distefano, Peter O’Hearn, and Hongseok Yang. 2009. Compositional shape analysis by means of bi-abduction. In ACM SIGPLAN Notices, Vol. 44. ACM, 289-300.

[10] Pavol Černỳ, Thomas A Henzinger, Arjun Radhakrishna, Leonid Ryzhyk, and Thorsten Tarrach. 2013. Efficient synthesis for concurrency by semantics-preserving transformations. In International Conference on Computer Aided Verification. Springer, 951-967.

[11] Sigmund Cherem, Trishul Chilimbi, and Sumit Gulwani. 2008. Inferring locks for atomic sections. In SIGPLAN Conference on Programming Language Design and Implementation (PLDI). ACM, New York, NY, USA, 304-315. https://doi.org/10.1145/1375581.1375619

[12] Leonardo De Moura and Nikolaj Bjørner. 2008. Z3: An efficient SMT solver. Tools and Algorithms for the Construction and Analysis of Systems (2008), 337-340.

[13] Dongdong Deng, Wei Zhang, and Shan Lu. 2013. Efficient concurrencybug detection across inputs. In Acm Sigplan Notices, Vol. 48. ACM, 785-802.

[14] Edsger W Dijkstra. 1971. Hierarchical ordering of sequential processes. In The origin of concurrent programming. Springer, 198-227.

[15] Edsger W. Dijkstra. 2002. Cooperating Sequential Processes. Springer New York, New York, NY, 65-138. https://doi.org/10.1007/ 978-1-4757-3472-0_2

[16] Isil Dillig and Thomas Dillig. 2013. Explain: a tool for performing abductive inference. In International Conference on Computer Aided Verification. Springer, 684-689.

[17] Isil Dillig, Thomas Dillig, Boyang Li, and Ken McMillan. 2013. Inductive invariant generation via abductive inference. In Acm Sigplan Notices, Vol. 48. ACM, 443-456.

[18] Isil Dillig, Thomas Dillig, Boyang Li, Ken McMillan, and Mooly Sagiv. 2017. Synthesis of circular compositional program proofs via abduction. International fournal on Software Tools for Technology Transfer 19, 5 (2017), 535-547.

[19] E Allen Emerson and Roopsha Samanta. 2011. An Algorithmic Framework for Synthesis of Concurrent Programs.. In ATVA. Springer, 522530.

[20] Michael Emmi, Jeffrey S. Fischer, Ranjit Jhala, and Rupak Majumdar. 2007. Lock allocation. In POPL '07: Proc. 34th symposium on Principles of Programming Languages. ACM Press, 291-296.

[21] Kostas Ferles, Jacob Van Geffen, Isil Dillig, and Yannis Smaragdakis. 2018. Symbolic Reasoning for Automatic Signal Placement (Extended Version). (2018). arXiv:1804.02503

[22] Cormac Flanagan and K Rustan M Leino. 2001. Houdini, an annotation assistant for ESC/Java. In International Symposium of Formal Methods Europe. Springer, 500-517.

[23] Rui Gu, Guoliang Jin, Linhai Song, Linjie Zhu, and Shan Lu. 2015. What change history tells us about thread synchronization. In Proceedings of the 2015 10th Joint Meeting on Foundations of Software Engineering. ACM, 426-438.

[24] Per Brinch Hansen. 1973. Operating system principles. Prentice-Hall, Inc. 
[25] Tim Harris and Keir Fraser. 2003. Language support for lightweight transactions. In OOPSLA '03: Proc. 18th conf. on Object-oriented Programing, Systems, Languages, and Applications. ACM Press, Anaheim, CA, 388-402. https://doi.org/10.1145/949305.949340

[26] Maurice Herlihy and J. Eliot B. Moss. 1993. Transactional Memory: Architectural Support for Lock-Free Data Structures. In Proceedings of the 20th Annual International Symposium on Computer Architecture. 289-300.

[27] Michael Hicks, Jeffrey S. Foster, and Polyvios Prattikakis. 2006. Lock Inference for Atomic Sections. In Proceedings of the First ACM SIGPLAN Workshop on Languages, Compilers, and Hardware Support for Transactional Computing.

[28] C. A. R. Hoare. 1971. Towards a theory of parallel programming. In International Seminar on Operating System Techniques.

[29] Charles Antony Richard Hoare. 1974. Monitors: An operating system structuring concept. In The origin of concurrent programming. Springer, 272-294.

[30] David Hovemeyer and William Pugh. 2004. Finding concurrency bugs in Java. In Proc. of PODC, Vol. 4.

[31] Wei-Lun Hung and Vijay K. Garg. 2013. AutoSynch: An Automaticsignal Monitor Based on Predicate Tagging. In Proceedings of the 34th ACM SIGPLAN Conference on Programming Language Design and Implementation (PLDI '13). ACM, New York, NY, USA, 253-262. https://doi.org/10.1145/2491956.2462175

[32] Guoliang Jin, Wei Zhang, Dongdong Deng, Ben Liblit, and Shan Lu. 2012. Automated Concurrency-Bug Fixing.. In OSDI, Vol. 12. 221-236.

[33] Pallavi Joshi, Mayur Naik, Chang-Seo Park, and Koushik Sen. 2009. CalFuzzer: An extensible active testing framework for concurrent programs. In Computer Aided Verification. Springer, 675-681.

[34] Pallavi Joshi, Mayur Naik, Koushik Sen, and David Gay. 2010. An effective dynamic analysis for detecting generalized deadlocks. In Proceedings of the eighteenth ACM SIGSOFT international symposium on Foundations of software engineering. ACM, 327-336.

[35] Vineet Kahlon. 2012. Automatic lock insertion in concurrent programs. In Formal Methods in Computer-Aided Design (FMCAD), 2012. IEEE, 16-23.

[36] Terence Kelly, Yin Wang, Stéphane Lafortune, and Scott Mahlke. 2009. Eliminating concurrency bugs with control engineering. Computer 42 , 12 (2009).

[37] Sepideh Khoshnood, Markus Kusano, and Chao Wang. 2015. ConcBugAssist: constraint solving for diagnosis and repair of concurrency bugs. In Proceedings of the 2015 International Symposium on Software Testing and Analysis. ACM, 165-176.

[38] Shuvendu K Lahiri and Shaz Qadeer. 2009. Complexity and Algorithms for Monomial and Clausal Predicate Abstraction.. In CADE. Springer,
214-229.

[39] James R. Larus and Ravi Rajwar. 2007. Transactional Memory. Morgan \& Claypool.

[40] Doug Lea. 1999. Concurrent Programming in Java. Second Edition: Design Principles and Patterns (2nd ed.). Addison-Wesley.

[41] Brandon Lucia and Luis Ceze. 2009. Finding concurrency bugs with context-aware communication graphs. In Proceedings of the 42nd Annual IEEE/ACM International Symposium on Microarchitecture. ACM, 553-563.

[42] Bill McCloskey, Feng Zhou, David Gay, and Eric Brewer. 2006. Autolocker: Synchronization inference for atomic sections. In POPL '06: Proc. 33rd symposium on Principles of Programming Languages. ACM Press, 346-358. https://doi.org/10.1145/1111037.1111068

[43] Madanlal Musuvathi, Shaz Qadeer, Thomas Ball, Gerard Basler, Piramanayagam Arumuga Nainar, and Iulian Neamtiu. 2008. Finding and Reproducing Heisenbugs in Concurrent Programs.. In OSDI, Vol. 8. $267-280$.

[44] Mayur Naik, Alex Aiken, and John Whaley. 2006. Effective static race detection for Java. Vol. 41. ACM.

[45] Aleksey Shipilev, Sergey Kuksenko, Anders Astrand, Staffan Freiberg, and Henrik Loef. [n. d.]. OpenJDK: JMH. ([n. d.]). http://openjdk.java. net/projects/code-tools/jmh/

[46] Yannis Smaragdakis, Jacob Evans, Caitlin Sadowski, Jaeheon Yi, and Cormac Flanagan. 2012. Sound Predictive Race Detection in Polynomial Time. In ACM Symposium on Principles of Programming Languages (POPL). ACM Press, 387-400.

[47] Raja Vallée-Rai, Phong Co, Etienne Gagnon, Laurie Hendren, Patrick Lam, and Vijay Sundaresan. 1999. Soot-a Java bytecode optimization framework. In Proceedings of the 1999 conference of the Centre for Advanced Studies on Collaborative research. IBM Press, 13.

[48] Martin Vechev, Eran Yahav, and Greta Yorsh. 2010. Abstraction-guided Synthesis of Synchronization. In Proceedings of the 37th Annual ACM SIGPLAN-SIGACT Symposium on Principles of Programming Languages (POPL '10). ACM, New York, NY, USA, 327-338. https://doi.org/10. $1145 / 1706299.1706338$

[49] Yin Wang, Stéphane Lafortune, Terence Kelly, Manjunath Kudlur, and Scott Mahlke. 2009. The theory of deadlock avoidance via discrete control. In ACM SIGPLAN Notices, Vol. 44. ACM, 252-263.

[50] Weiwei Xiong, Soyeon Park, Jiaqi Zhang, Yuanyuan Zhou, and Zhiqiang Ma. 2010. Ad Hoc Synchronization Considered Harmful.. In OSDI, Vol. 10. 163-176.

[51] Haiyan Zhu, Thomas Dillig, and Isil Dillig. 2013. Automated inference of library specifications for source-sink property verification. In Asian Symposium on Programming Languages and Systems. Springer, 290306. 